\title{
A Nodal Electricity Market Design Based on Regulated Access to Transmission Network
}

\author{
Jean Constantinescu \\ ROENP INIT SRL, Bucharest 010537, Romania
}

Received: May 06, 2013 / Accepted: June 20, 2013 / Published: December 31, 2013.

\begin{abstract}
Accomodation of power system constraints with the market mechanism is encountered as a major challenge along the way toward implementation of different electricity market designs. Allocation of fixe or flow-dependent inter-zone trading capacities by the PX (power exchange) can not be accepted unreservedly. The paper is meant to show that a nodal electricity market design that is based on bids for local energy and a regulated transmission access including allocation of "entry-exit" transmission capacity would be the desired solution. The market players could easily optimize their portfolio while the TSOs (transmission system operators) are requested to mobilize the network's in-built flexibility to increase the cross zonal capacity. In the proposed market design, the PX's allocation of trading capacity is clearly separate from the TSO's management of the power system operational constraints. Clear operator roles would enlarge access to electricity market as well as market integration of variable RESs (renewable energy sources) that are critically dependent on short notice access to regional markets.
\end{abstract}

Key words: Electricity market design, nodal model, network access, transmission capacity, congestion management.

\section{Introduction}

In accordance with the EU directive of electricity market, the power transmission service is defined and priced in relation to "entry" and "exit" grid nodes. But, in the $\mathrm{CB}$ (cross-border) trade particularly, the transmission service is conditional upon the power flow path across the network. The service customer may be expected to participate in the invitation to additional tender for cross-border trading capacity, with an impact on transaction volume and price. Instead of being limited themselves to implicit allocation of "entry-exit" grid capacities, the PXs (power exchanges) are requested to relieve transmission constraints across the network based on auctions of so-called cross zonal capacity available for trade. However, this capacity essentially varies with the PS (power system) state, while TSOs (transmission system operators) only are in the right position to assess

Corresponding author: Jean Constantinescu, Dr. Eng., research fields: power system and electricity market. E-mail: jeanconstantinescu@ymail.com. it and relief the constraints cost-efficiently, as shown in this paper. Coupling of the North Western European day-ahead market experiences delay and problems due to the loop flow issue in spite of the "flow-based" model of capacity calculation and allocation.

NP (nodal pricing) is an approach to the design of electricity market that avoids auction for $\mathrm{CB}$ transmission capacity. Essentially, the NP is a constrained economic dispatch that selects the bids to buy and sell electricity at network nodes, and determines the price-taking MW (active power) schedules between those nodes. There is a large literature on NP; the published works [1, 2] contain extensive reference to this literature. NP was introduced in New Zealand (1997), some US markets (PJM 1998, New York 1998, New England 2003) and Great Britain (2005) with the aim to have the real market clearing price discovered, which is different at each network node. Transmission cost is settled at the difference in nodal prices. The (linearized, d.c.) model of PS operational constraints is the weak spot of the 
current approaches. It is actually an only rough approximation of the real PS operational constraints that neglects the system's stability condition.

The paper presents an alternative solution for accomodation the PS constraints with the nodal market mechanism. TC (transmission capacity) made available to the market is also node-related; it would be allocated implicitly by the market mechanism and traded at a regulated nodal price. TC allocation becomes clearly separated from the TSO's TM (transmission monitoring) as PS constraint identification and relief. And, TM only is based on the control of power transfer across the relevant interconnections.

\section{Regulated Transmission Rate}

The principle of fair cost allocation requires the TSO to charge transmission customers according to the impact of service on the total cost of transmission, as reflected by the MC (marginal cost) of transmission. It is currently symbolized by $\mathrm{G}$ for a generation injection and by $\mathrm{L}$ for a load injection. The MC arises either as by-information in PS optimal dispatch or can be estimated on the basis of coefficients for loss and congestion multiplied by the prices in markets for electricity and balance energy, respectively [3]. To meet the transmission RR (revenue requirement), a MOF (market opportunity fee) would overcharge the G and L as a pro-rata with the benefit that grid user gets from access to the network [4]. The ex-post MOFs do not alter the regulated nature of the transmission rates since they are rigorously adjusted to the regulated RR. In a multi-TSO regulatory environment that is harmonized, the Gs and Ls are supposed to be established following the same rules of grid service, grid configuration and cost definition. An ITC (Inter-TSO compensation) mechanism would integrate all the cost effects of transits, including the congestion cost.

\section{Nodal Transmission Capacity}

TC is a threshhold for the volume of electricity transactions that need access to transmission network.
Accordingly, CM (congestion management) is the control of transactions as against TC in view of protecting integrity of the PS operation. TC makes clear sense for the market players as grid users if it expresses the DP (driving-point) or "point-of-connection" power transfer capability of the network [5] (Fig. 1).

DP-TC reflecting a variety of PS operational constraints is, in general, essential data for a connection permit. Dimo demonstrated that security requirements of the PS can be determined for every network node [6].

For CB trade, however, the specific European electricity market regulation [7] defines the TC as a PP (point-to-point) power transfer capability. That is, the interconnector only appears responsible for the PS operational limit. The DP and PP transmission capacity models express the key requirements of grid access and market design differently, namely:

Meaningfulness of transmission capacity. While the DP-TC information is fairly easy to understand, allowing a market player to optimize its market portfolio, this can not be said of PP-TC capacity that does not appeal to the average grid user;

Predictability of transmission capacity. While a point-of-connection capacity is relatively stable, the physical limits of interconnectors are generally volatile even on a monthly basis. Full predictability of capacity at the point of network connection must be a defining feature of future smart grids;

Increase in network efficiency and market integration. DP capacity makes a natural model for implicit allocation of grid capacity. Allocation of PP capacity to market players in explicit auctions does not incentivize TSOs to allow greater utilization of the transmission system;

Consistent network planning and usage. DP capacity reflects the transmission capability in network planning. If standards of planning and operation were not the same, electricity transactions might either jeopardize PS integrity or be excessively limited. 


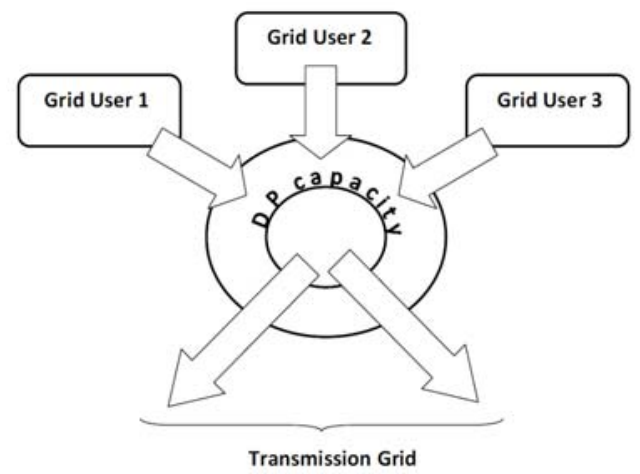

Fig. 1 Nodal (DP, point-of-connection, “entry-exit”) model of access to transmission network.

\section{Transmission Monitoring}

\subsection{Transmission Monitoring as PS Constraint} Management

According to the operational rules, the TSO manages the PS constraints through the standard decision making process (Fig. 2), namely:

PS schedule after receipt of transaction notifications, and real-time dispatching operation, which includes correction of PS schedule when deviations from the system normal operation are detected in the next few hours.

TM identifies the PS operational constraints and establishes the remedial measures in both day-ahead and real-time TSO's operations. Time and action are inherently different for transmission access in market mechanism, on one hand, and system operation on the other. Typically, the ante-nomination CM for the purpose of TC compliance may lead to adjustment of active power balance of the source and sink network zones. By contrast, the TSO's out-of-market operations mobilize a broader range of generation-/transmission network-/load-based countermeasures in order to both adjust the area power balances and enlarge the constrained TLRI (transmission limit of relevant interconnection).

The next exercise that was carried out under the SEETSOC project [8] is meant to indicatively rend evident a step-wise TM process as PS constraint management. ROENP was a member of international consortium to the SEETSOC that received financing from the FP7 of the European Union.

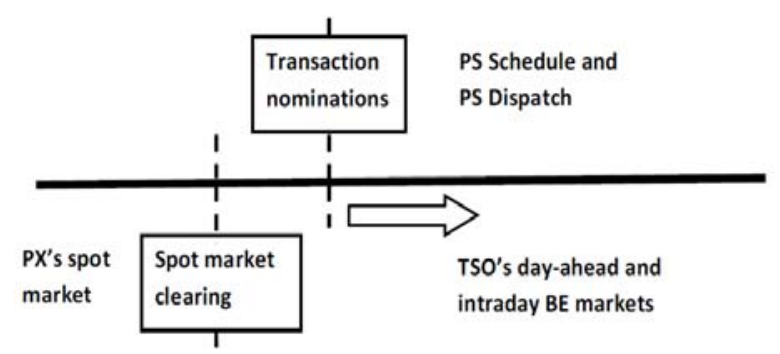

Fig. 2 Sequence of centralized market deals and TSO's operations.

\subsection{TM Study Case}

\subsubsection{Model Preparation}

A hypothetic redispatch scenario that renders evident the system's maximal loadability and the relevant interconnections is prepared. To be conservative, the PS limit state should be the result of the most severe worsening of system operation. To this aim, the generators are ranked according to their impact on the system stability as defined by the SCRI (stability criterion of relative impact). The SCRI is calculated as relative synchronizing power $(\partial P / \partial \delta)^{*}$ on the simplest equivalent model for each network node with generators [9]. On the basis of ordered SCRI list, the load of generators which improve the stability is decreased while that of generators with a negative impact on the stability is increased. For the 9-machine 32-node power system (Fig. 3), the load of generators M6 and M8 with the least SCRI was incrementally brought up while that of generators M1, M2, M3, M4 and M7 with larger SCRIs was simultaneously backed down.

Four areas (A, B, C, D) with the corresponding area border cut-sections were identified by grouping generating units in conformity with the sense of their output change.

\subsubsection{Constraint Identification}

A number of N-1 network contingencies as unplanned line disconnections and generator outages were simulated, as well. SAMI software ${ }^{1}$ determined

\footnotetext{
${ }^{1}$ SAMI software was developed with the intention to prevent numerical instability as well as idealistic representation of the nodal power injections in the conventional load flow models. It was widely used in Romanian TSO operations for determination of the network's power transfer capabilities as either limits of node power injections or TLRIs [9].
} 


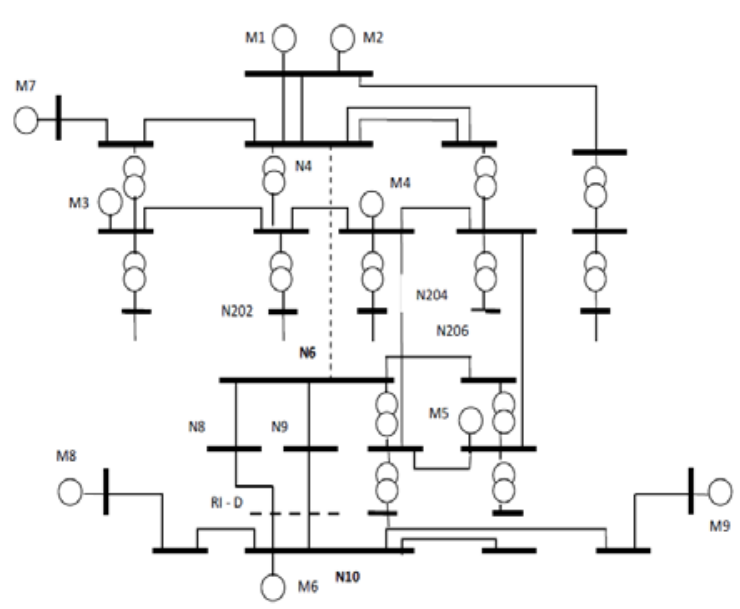

Fig. 3 CIGRE TF 38-02-08 test network.

the trajectories that are followed by the node voltages during the step-by-step redispatch scenario, and corresponding line flows up to the critical PS state. The maximal power transfers are calculated for the state that precedes PS instability. The stability is checked up with an ISSM (integral steady-state stability margin) index, ranging from 0 to 1 irrespective of the PS size. The TLRIs were established for each relevant interconnection by subtracting the TRMs from the corresponding stability-based limit flows. Typically, the TRM can be chosen at $20 \%$ of the limit flow under PS normal operation. For the interconnection D (N8-N10; N9-N10), the lowest TLRI 20 is 1,606.2 MW. It does not comply with 1,737.9 $\mathrm{MW}$, an exchange schedule resulting from transaction nominations. To relieve the interconnection's overschedule (165 MW for $20 \%$ reserve), cost-efficient remedial measures must be found.

\subsubsection{Constraint Relief Strategy}

The TSO attempts to both diminish the flow schedule on the interconnection, by generation redispatch, and increase the D-TLRI by increasing the system's stability margin.

First, a cost-efficient generation redispatch amounting to $165 \mathrm{MW}^{2}$ is found. The suppliers of balance energy

\footnotetext{
2 The total generation redispatch equals the interconnection's overshedule since no loop flows can occur. In general, there might be applied a less than 1 PTDF (power transfer distribution factor) as a fraction of the amount of a transaction from one zone to another.
}

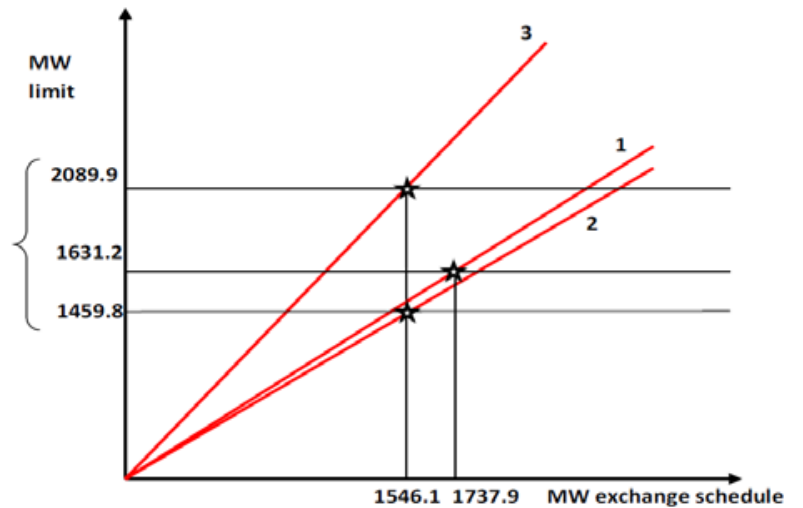

Fig. 4 Exchange flows and limits for interconnection D following (1) transaction nominations; (2) MW redispatch and (3) MW redispatch and voltage regulation.

are selected on the basis of stability impact coefficients (SCRIs) and energy prices (BEPS), separately for up-ward and down-ward regulation. $\Sigma_{i} S C R I_{i} * B E P_{i}$ are minimal when the loads of $\mathrm{M} 1$ and $\mathrm{M} 2$ are increased by $100 \mathrm{MW}$ and $65 \mathrm{MW}$, respectively, while the M6 load is decreased by 165 MW. However, the active power rebalance on a "source-to-sink" model diminishes the D-TLRI as well, from 1,631.2 MW to 1,459.8 MW; it proves to be insufficient as can be noticed in Fig. 4 (Line 2).

Second, complementary remedies with an impact on the network's voltage level, on the PS stability implicitly, are necessary to remove the interconnection overschedule. Two options were found in this exercise: either disconnection of shunt reactors at the nodes N202, N204 and N206 (Line 3 in Fig. 4), or re-establishment of M8's $\mathrm{AVR}^{3}$ that was initially out of service.

\section{Conclusions}

Power flow bottlenecks tend to become chronic when trading cross zonal transmission capacity is allocated either implicitly, as MW rebalance by PX, or explicitly, by separate capacity auctioning. With existing CAO and market coupling models, the access is not reached for full network capacity because the cross zonal NTCs depends on the TSO's operations. And, the network's

\footnotetext{
${ }^{3}$ This is modelled as a reduction of the transient synchronous reactance that is used for calculation of the ISSM.
} 
in-built flexibility that can be mobilized by the TSO is ignored. A PX is not able to determine real PS operational constraints and find solutions to enlarge the access to electricity market. Regulation of access to the power transmission network on the basis of "entry-exit" transmission capacity and rate is an ideal solution for a PX to sell energy all at once with release of transmission capacity. Clear separation between PX's congestion management as TC allocation and TSO's TM as management of the power system operational constraints after completion of electric market participants' auto scheduling can enlarge access to electricity market, avoid market fragmentation and mitigate power system operational risk as well. PS constraint management is significantly more complex than commercial $\mathrm{CM}$, and includes purchase of streamlined balance energy and reactive support from the market providers with the largest positive impact on PS stability.

In general, a nodal market design based on consistent definition of transmission capacity in network planning and network usage would yield significant advantages, as follows:

Increased market liquidity because the transactions will migrate to centralized markets when electricity is sold all at once with the release of network capacity;

Reliable use and sustainable development of the power network since the TSOs would become really interested to increase interconnection capacity made available to the market;

Predictable network capacity at the user connection point, as a desirable smart grid feature and requirement for integration of the variable RESs.

\section{References}

[1] S.S. Oren, P.T. Spiller, P. Varaiya, F. Wu, Nodal prices and transmission rights: A critical appraisal, The Electricity Journal 8 (3) (1995) 24-35.

[2] F. Leuthold, I. Rumiantseva, H. Weigt, T. Jeske, C. Hirschhausen, Nodal pricing in the German electricity sector-A welfare economics analysis, with particular reference to implementing offshore wind capacities [Online], Dresden, 2005, http://ssrn.com/abstract=1137382.

[3] J. Constantinescu, Electricity Market Design Based on a Nodal Model of Access to the Network, Bucharest, 2010, pp. 10-11.

[4] J. Constantinescu, An electricity market mechanism based on benefit-balanced transmission rates, VGB PowerTech 03 (2010) 93-95.

[5] J. Constantinescu, A Unitary Approach of Operational Security and Market Design in a Multi-TSO Interconnection, CIGRE report C5-303, 2008.

[6] P. Dimo, Nodal Analysis of Power Systems, Abacus Press, Tunbridge Wells, Kent, 1975.

[7] European Parliament and Council of the European Union, Regulation on Conditions for Access to the Network for Cross-Border Exchanges in Electricity and Repealing Regulation (EC) No. 1228, 2003.

[8] SEETSOC-EU-FP7 Project, South-East European TSO Challenges, report on Transportation Monitoring (WP5/D5.3.2), 2011.

[9] J. Constantinescu, Practical assessment of the power system stability margins, Rev. Roum. des Sci. Techn. 39 (2) (1994) 217-222. 